\title{
Successful treatment of COVID-19 with remdesivir in the absence of humoral immunity
}

\section{Matthew S Buckland}

Barts

James Galloway

Kings College Hospital

Caoimhe Nic Fhogartaig

Bart's Health

Luke Meredith

University of Cambridge

Nicholas M. Provine

University of Oxford

Stuart Bloor

University of Cambridge

Ane Ogbe

University of Cambridge

Wioleta M. Zelek

Systems Immunity Institute and Dementia Research Institute, Cardiff University, Cardiff

Anna Smielewska

Cambridge University Hospitals

Anna Yakovleva

University of Cambridge

Tiffeney Mann

University of Cambridge

Laura Bergamaschi

University of Cambridge

Lorinda Turner

University of Cambridge

Frederica Mescia

University of Cambridge

Erik J.M. Toonen

Hycult Biotech Ltd

Carl-Philipp Hackstein

University of Oxford

Hossain Delowar Akther 
University of Oxford

\section{Vinicius Adriano Vieira}

University of Oxford

\section{Lourdes Ceron-Gutierrez}

University of Cambridge

Jimstan Periselneris

University of Cambridge

\section{Sorena Kiani-Alikhan}

University of Cambridge

\section{Sofia Grigoriadou}

Barts Health

\section{Devan Vaghela}

Cambridge University Hospitals

\section{Sara E. Lear}

Cambridge University Hospitals

\section{Estee Torok}

University of Cambridge

\section{William L. Hamilton}

Cambridge University Hospitals

\section{Josh Quick}

University of Birmingham

Joanne Stockton

University of Birmingham

\section{Peter Nelson}

Belfast Health and Social Care Trust

\section{Michael Hunter}

Belfast Health and Social Care Trust

\section{Tanya I Coulter}

Belfast Health and Social Care Trust

\section{Lisa Devlin}

Belfast Health and Social Care Trust

\section{John Bradley}

University of Cambridge

\section{Ken Smith}

University of Cambridge

\section{Willem Ouwehand}

University of Cambridge

\section{Lise Estcourt}

University of Oxford 
Heli Harvala Simmonds

NHSBT

Dave Roberts

University of Oxford

lan Wilkinson

University of Oxford

Nick Screaton

Papworth Hospital

\section{Nick Loman}

University of Birmingham

Paul Lyons

University of Cambridge

Rainer Doffinger

University of Cambridge

Paul Morgan

University of Cardiff

lan Goodfellow

University of Cambridge

Paul Klenerman

University of Oxford

Paul Lehner

University of Cambridge

Nick Matheson

University of Cambridge

James Thaventhiran ( $\nabla$ jedt2@cam.ac.uk)

University of Cambridge

\section{Research Article}

Keywords: Remdesivir, SARS-CoV-2, COVID-19, XLA

Posted Date: September 23rd, 2020

DOl: https://doi.org/10.21203/rs.3.rs-79022/v1

License: (1) This work is licensed under a Creative Commons Attribution 4.0 International License. Read Full License

Version of Record: A version of this preprint was published at Nature Communications on September 23rd, 2020. See the published version at https://doi.org/10.1038/s41467-020-19761-2. 


\section{Abstract}

The response to the coronavirus disease 2019 (COVID-19) pandemic has been hampered by lack of an effective severe acute respiratory syndrome coronavirus 2 (SARS-CoV-2) antiviral therapy. Here we report the successful use of remdesivir in a patient with COVID-19 and the prototypic genetic antibody deficiency X-linked agammaglobulinaemia (XLA). Despite evidence of complement activation and a robust $T$ cell response, the patient developed persistent SARS-CoV-2 pneumonitis, without progressing to multi-organ involvement. His unusual clinical course identifies a key role for SARS-CoV-2 antibodies in both viral clearance and progression to severe disease. In the absence of these confounders, we took an experimental medicine approach to examine the in vivoutility of remdesivir. Over two independent courses of treatment, we observed a dramatic, temporally correlated clinical and virological response, leading to clinical resolution and viral clearance, with no evidence of acquired drug resistance. We therefore provide unambiguous evidence for the antiviral efficacy of remdesivir in vivo, and its potential benefit in selected patients.

\section{Introduction}

The prodrug nucleoside analogue remdesivir is a broad-spectrum antagonist of viral RNA-dependent RNA polymerase (RdRp) enzymes, leading to inhibition of SARS-CoV-2 replication in vitro ${ }^{1,2,3}$ and pre-clinical benefit in a macaque model of COVID-19. ${ }^{4}$ Two recent RCTs have tested the efficacy of remdesivir in patients. The first was underpowered, and failed to show clinical benefit. ${ }^{5}$ Preliminary data from the second showed a statistically significant reduction in illness duration, and a trend to reduced mortality. ${ }^{6}$ No convincing evidence of virological efficacy was reported in either study.

Whilst RCTs provide the gold-standard for evaluation of the efficacy of new therapeutic interventions, comparing the average responses of patients in heterogeneous treatment and control groups may mask the potential benefits for individual patients. Evaluation of therapeutics for COVID-19 is particularly complicated by the highly variable clinical course. Furthermore, as well as mediating clearance of SARSCoV-2, the immune response may also contribute to severe COVID-19 pathology, independent of viral replication.

It is therefore unclear whether the limited response to remdesivir observed in RCTs reflects inadequate in vivo antiviral activity, or the need for concurrent immunomodulation. To minimise heterogeneity attributable to the immune response, we therefore take a reductionist, experimental medicine approach to evaluate the efficacy of remdesivir for treatment of COVID-19 in vivo, by studying a rare patient in whom the contribution of humoral (antibody-dependent) immunity to viral clearance and immunopathology is controlled genetically by the primary immunodeficiency XLA.

\section{Results}


$\mathrm{XLA}$ is caused by mutations in the gene encoding Bruton's tyrosine kinase $(B T K)$, leading to an absence of mature $B$ lymphocytes and immunoglobulins (antibodies). The subject of this study is a 31 year old man with XLA, whose past medical history is summarised in the Methods. His illness began with fever, cough, nausea and vomiting. On day 19 , a nasopharyngeal/throat swab was positive for SARS-CoV-2 RNA, and he commenced treatment with hydroxychloroquine and azithromycin (Supplementary Figure 1). His symptoms persisted, and a repeat nasopharyngeal/throat swab on day 28 remained positive for SARS-CoV-2 RNA.

The patient was admitted to hospital on day 30 because of worsening dyspnea, and supplemental oxygen was commenced (Fig. 1a). Blood tests showed a rising CRP and mild lymphopaenia, elevated IL6, IL-10, TNF-a and IFN-g, but normal D-Dimer and troponin, with no evidence of significant coagulopathy, renal or liver dysfunction (Fig. 1 and Tables 1-4). Blood and sputum cultures for bacteria remained negative throughout the course of the illness, procalcitonin was repeatedly normal, and two trials of broad-spectrum intravenous antibiotics had no improved effect (Supplementary Figure 1). A sputum sample remained positive for SARS-CoV-2 RNA, and a CT chest scan showed widespread patchy groundglass opacity in the lower lobes, consistent with COVID-19 pneumonitis (Fig. 1b).

Immunocompetent adults with severe COVID-19 typically exhibit a monophasic acute illness, with hospital admission and progressive respiratory failure 7-10 days after symptom onset ${ }^{7,8}$. In contrast, our patient exhibited a very unusual pattern of SARS-CoV-2 infection, with persistent fever and pneumonitis for $>30$ days, but without progression to acute respiratory distress syndrome (ARDS) or multi-organ involvement. This relatively stable baseline allowed the detailed assessment of clinical, virological and immune responses during two independent challenges with remdesivir.

\section{Clinical response to remdesivir}

On day 34, hydroxychloroquine and azithromycin were discontinued, and the patient commenced a 10 day course of remdesivir. His fever and dyspnea improved within 36 hours of the first dose, nausea and vomiting ceased, and rising oxygen saturation allowed discontinuation of supplemental oxygen. This dramatic clinical response was accompanied by a progressive decrease in CRP, a rise in total lymphocyte count, and an improvement in ground-glass opacification on repeat CT chest (Fig. 1a-b). The patient was therefore discharged on day 43.

7 days after discharge, his fever, dyspnea and nausea returned. He was readmitted to hospital on day 54 , and supplemental oxygen was commenced. A further sputum sample was positive for SARS-CoV-2 RNA, and a CT pulmonary angiogram showed evidence of ongoing pneumonitis (Fig. 1a-b). Consistent with a recrudescence of COVID-19, the patient's CRP increased, and his lymphocyte count fell. On day 61, he therefore began treatment with a further 10 day course of remdesivir. Once again, his symptoms rapidly improved, his fever and requirement for supplemental oxygen resolved, and his CRP and lymphocyte count normalised (Fig. 1a). 
The patient therefore exhibited a dramatic clinical response, tightly correlated with the administration of remdesivir, over two independent challenges. Because of his underlying immunodeficiency, protracted illness, and relapse after the first course of remdesivir, he was further treated with 2 units of convalescent plasma on days 69 and 70, to provide secondary prophylaxis against SARS-CoV-2 infection (Fig. 1a). He was discharged 3 days later, and has remained apyrexial and asymptomatic over a further 28 days of follow-up.

\section{Virological response to remdesivir}

SARS-CoV-2 RNA was identified on nasopharyngeal/throat swabs early in the patient's illness, but became undetectable on these samples from day 36 (Fig. 1a). Because of his underlying bronchiectasis, the patient habitually expectorates small volumes of sputum. SARS-CoV-2 RNA was readily detectable in these samples until day 64, 4 days into his second remdesivir course, allowing non-invasive monitoring of his lower respiratory tract. Samples from blood, urine, faeces and a rectal swab were all negative.

Strikingly, levels of SARS-CoV-2 RNA in sputum fell progressively during the patient's first course of remdesivir, corresponding with his clinical response (Fig. 1a). Nonetheless, SARS-CoV-2 RNA remained detectable at low levels. Upon cessation of remdesivir treatment, levels of SARS-CoV-2 RNA increased again in parallel with the recrudescence of symptoms. The effect of the second course of remdesivir was even more rapid and complete, with SARS-CoV-2 RNA becoming undetectable after 4 days (Fig. 1a). The patient therefore exhibited a dramatic virological response, tightly correlated with both the administration of remdesivir and resolution of his symptoms.

To confirm that the recurrent detection of SARS-CoV-2 RNA reflected viral persistence, rather than reinfection, isolates were sequenced regularly over the course of the patient's illness (Fig. 1a and Supplementary Figure 2 and Table 5). All isolates belonged to the B2.6 lineage, a very uncommon lineage globally with only 47 isolates in total from the UK, making reinfection extremely unlikely. Compared with the Wuhan-Hu-1 reference sequence, 17 single nucleotide polymorphisms (SNPs) were present in at least one isolate (Supplementary Figure 2). Amongst these, the only SNP in the remdesivir target gene $n s p 12$, encoding the RdRp, was a synonymous variant (C14805T), present at equivalent abundance across all isolates. Despite the combination of underlying immunodeficiency, prolonged viral shedding and repetitive treatment, there was therefore no evidence of acquired remdesivir resistance.

\section{Contribution of antibodies to clearance of SARS-CoV-2}

$\mathrm{XLA}$ is the prototypic genetic disorder of the humoral immune system, and the most obvious explanation for our patient's failure to clear his infection spontaneously is his lack of antibodies to SARS-CoV-2. Patients with XLA are known to be at risk from persistent RNA viral infections, particularly chronic enteroviral meningoencephalitis ${ }^{9}$. This risk is mitigated by immunoglobulin replacement therapy, including antibodies to enteroviruses. ${ }^{10}$ Conversely, available pooled immunoglobulin preparations antedate the COVID-19 pandemic, and lack specific SARS-CoV-2 antibodies. 
We therefore assessed the humoral immune response to SARS-CoV-2 in our patient. As expected, despite maintenance of regular immunoglobulin replacement, antibodies to SARS-CoV-2 spike and nucleocapsid proteins were undetectable by immunoassay prior to treatment with convalescent plasma (Fig. 2a-b), and no neutralisation activity was observed against lentiviral particles pseudotyped with SARS-CoV-2 spike protein (Fig. 2c). Following administration of convalescent plasma, antibody levels and neutralisation activity rose commensurately (Fig. 2a-c).

\section{$\underline{\mathrm{CD} 8+\mathrm{T} \text { cell response to SARS-CoV-2 }}$}

Together with CD8+ T cells, antibodies are known to contribute to the control of other RNA viruses, including HIV ${ }^{11}$, Hepatitis $C^{12}$ and LCMV infection of mice ${ }^{13,14}$. Two recent reports of patients with XLA and COVID-1915, 16 did not describe persistent clinical disease. This suggests that, similar to the LCMV model, CD8+ T cell immunity can sometimes compensate for humoral deficiency in the control SARSCoV-2. We therefore assessed the antigen-specific CD8+ T cell response to SARS-CoV-2 in our patient.

Immunodominant CD8+ T cell epitopes of SARS-CoV- $2^{17}$ and other coronaviruses ${ }^{18,19}$ are contained within the spike protein. We first measured the quality of the CD8+ T cell response by flow cytometry for antigen-stimulated effector protein expression ${ }^{20}$ (Fig. 3a). The frequency of spike-specific CD $8+T$ cells within the circulation was comparable to age-matched health care workers with acute COVID-19, trending upwards over the course of infection. Increasing polyfunctional effector capacity was evident from day 62 (Fig. 3b), particularly the appearance of TNF-a and IFN-g producing CD8+ T cells (Supplementary Figure 3).

We further assessed the coverage of the patient's antigen-specific response by measuring CD8+ T cell proliferation to peptide pools covering the S1, S2, M, N, ORF3, ORF6, ORF8 and ORF7 SARS-CoV-2 proteins, following viral clearance. At days 77 and 83 , the patient had striking responses to all viral peptides relative to age-matched COVID-19 infected HCWs (Fig. 3c-d), likely reflecting the prolonged duration of antigen exposure. Taken together, these data confirm the presence of a robust CD8+ T cell response to SARS-CoV-2. Whilst insufficient to resolve the infection spontaneously, this likely contributed to the clearance of virus during the second course of remdesivir.

\section{Role of antibodies in immunopathogenesis of COVID-19}

As well as their role in the anti-viral response, antibodies have the potential to cause immune pathology ${ }^{21}$. In a retrospective study of patients with SARS-CoV, the development of neutralising antibodies early in infection correlated with worse disease outcome ${ }^{22}$, and high antibody titres are associated with severe disease in COVID-1923. Our patient's lack of SARS-CoV-2 antibodies may therefore explain why, despite his persistent infection, he did not progress to ARDS or multi-organ involvement.

Antigen-antibody complexes are able to activate the classical complement pathway, with release of the anaphylatoxins $\mathrm{C} 3 \mathrm{a}$ and $\mathrm{C} 5 \mathrm{a}$ and formation of the multi-subunit terminal complement complex (TCC). C3-deficiency reduces lung pathology in a mouse model of SARS-CoV ${ }^{24}$, complement activation is a 
feature of COVID-1925, and deposition of TCC is observed in the microvasculature of patients with severe disease ${ }^{26}$.

We therefore assessed the level of complement activation in our patient. Despite the lack of disease progression, levels of C3a C3c, C5a and TCC were markedly elevated, comparable to levels seen in COVID19 patients admitted to the intensive care unit (Fig. 4a-d). Whilst complement activation correlates with and may be required for the development of ARDS and/or multi-organ failure, complement activation alone is therefore insufficient for disease progression, and the classical pathway is not required for complement activation in COVID-19.

\section{Discussion}

Taken together, our observations confirm that remdesivir is a potent antiviral agent for treating SARS-CoV2 infection in vivo. It is striking that previous reports from immunocompetent patients have generally not demonstrated the profound clinical and virological responses observed in this study. This is likely because, amongst the hospitalised patients included in $\mathrm{RCTs}^{5,6}$ or compassionate use programmes ${ }^{27}$ to date, progression or resolution of disease has been determined not by the level of SARS-CoV-2 replication, but by the evolution of immune pathology. Whilst remdesivir was given chronologically late in our patient's disease, he had not developed ARDS or multi-organ involvement. The utility of remdesivir in immunocompetent patients may therefore be maximised by early treatment in the most at risk, or by combination with targeted immunomodulatory therapies in the most sick.

The persistent disease observed in this patient strongly suggests that antibodies contribute to the control of SARS-CoV-2. Conversely, the lack of disease progression suggests that they also contribute to immune pathology in COVID-19. Aside from activating complement, antibodies may interact with Fc receptors on immune cells to drive macrophage activation and inflammatory cytokine production ${ }^{28}$. Strategies which target these interactions include plasmapheresis, or blockade of Fc receptors by saturating doses of intravenous immunoglobulin (IVIG). The potential for both beneficial and deleterious effects of SARSCoV-2 antibodies revealed by our study suggests that these interventions, like the administration of convalescent plasma, should be tailored to individuals or subgroups of patients with distinct clinical characteristics.

\section{Methods}

\section{Oversight}

The study was approved by the East of England - Cambridge South national institutional ethics review board (17/EE/0025). The patient provided written informed consent. Additional healthy controls, patients and healthcare workers with COVID-19 were enrolled to the NIHR BioResource Centre Cambridge (17/EE/0025) and the Oxford Gastrointestinal Illness Biobank (16/YH/0247). 


\section{Patient characteristics}

The subject of this study is a 31 year old man, born in the UK to parents of Pakistani origin, and diagnosed with XLA at the age of 12 . He has a family history of XLA affecting his brother, 4 maternal uncles and a male maternal cousin. Following investigation for recurrent chest infections, he was found to lack mature B cells and circulating immunoglobulins, and confirmed to have inherited the familial $\mathrm{c}$. 1430 delT mutation in the gene encoding BTK. Because of prior incomplete adherence to replacement immunoglobulin therapy, he developed middle lobe bronchiectasis.

\section{$\underline{\text { Remdesivir treatment }}$}

The first course of remdesivir was administered as part of a Gilead SIMPLE study (NCT04292899), the second course was provided as part of the Gilead Expanded Access Program (NCT04323761). In each case, the patient received an initial dose of $200 \mathrm{mg}$ IV, followed by 9 daily doses of $100 \mathrm{mg}$ IV (10 days total).

\section{Convalescent plasma}

Convalescent plasma was collected from individuals with previous, laboratory-confirmed SARS-CoV2infection at least 28 days after resolution of symptoms using established infrastructure and standard UK donor selection guidelines, as previously described ${ }^{29}$. Signed consent was obtained from each donor at the time of donation using NHS Blood and Transplant-approved consent forms, in accordance with Blood Safety and Quality Regulations enforced by the Medicines \& Healthcare products Regulatory Agency.

In brief, a total of at least $540 \mathrm{ml}$ of plasma (containing $<1 \times 10^{6}$ leucocytes per component) was collected via plasmapheresis from each donor, divided into two units, rapidly frozen and stored at $-25^{\circ} \mathrm{C}$. Donor blood samples were tested for SARS-CoV-2 RNA (Public Health England) and antibodies (EUROIMMUN (IgG) assay, PerkinElmer, London, UK). A signal to cut-off (S/CO) ratio of 9.1 in the EUROIMMUN assay was previously shown to identify donations with a neutralising antibody titre of $>l=1: 100$ in a SARS-CoV2 (isolate England/2020) microneutralisation assay with a specificity of $100 \% .{ }^{29}$ Both donations used in this study were collected in May 2020 and confirmed to be negative for SARS-CoV-2 RNA. EUROIMMUN S/CO ratios were 37.171 (first unit, $290 \mathrm{ml}$, administered on day 69) and 7.271 (second unit, 283ml, administered on day 70), respectively. For the second unit (lower S/CO ratio), the neutralising antibody titre was confirmed to be $>/=1: 100$ using the SARS-CoV-2 microneutralisation assay. ${ }^{29}$ Plasma was defrosted using a $37^{\circ} \mathrm{C}$ waterbath, and transfused within 4 hours of defrosting.

\section{Patient sampling}

Upper respiratory tract samples (nasopharyngeal/throat swabs) were collected in viral transport medium according to Public Health England (PHE) guidelines. Lower respiratory samples (sputum) were collected in universal containers and extracted following mucolysis with Mucolyse PL.701 sputum liquefying agent 
(Pro-Lab Diagnostics). To minimize RNA degradation, the time between sample collection and processing did not exceed $2 \mathrm{hr}$.

Peripheral blood mononuclear cells (PBMCs) were isolated using Leucosep tubes (Greiner Bio-One) with Histopaque-1077 (Sigma) by centrifugation at $800 \mathrm{~g}$ for 15 mins at room temperature. PBMCs at the interface were collected and rinsed twice with autoMACS running buffer (Miltenyi Biotech). All samples were processed within $4 \mathrm{hr}$ of venepuncture. Samples were then resuspended in freezing medium (FBS $90 \%$ supplemented with DMSO $10 \%$ ), frozen overnight at $-80^{\circ} \mathrm{C}$ in a Mr Frosty cell freezing containger (Nalgene) then transferred to liquid nitrogen for long term storage.

\section{SARS-CoV-2 molecular testing}

Sample testing was carried out using an in-house real time uniplex RT-PCR diagnostic assay for the detection of SARS-CoV-2 in the PHE Clinical Microbiology and Public Health Laboratory at Addenbrooke's Hospital, Cambridge. This assay targets a 222 base-pair region of the SARS-CoV- $2 n s p 12$ gene (encoding $\mathrm{RdRp})$, and has been validated for clinical use.

In brief, nucleic acid extraction was undertaken using the NUCLISENS easyMAG platform (Biomerieux, Marcy L-Etoile), in accordance with the manufacturer's instructions. Nucleic acids were extracted from $500 \mu \mathrm{L}$ sample, with a dilution of MS2 bacteriophage (4600pfu per extraction) added pre-extraction to act as an internal extraction and inhibition control. Molecular grade water was used as a negative control. Positive control material, BetaCoV/England/02/2020, was obtained from PHE Colindale (essentially, purified viral RNA diluted to give a cycle threshold (CT) value of 26-28).

The RdRp gene was detected using primers ATGGGTTGGGATTATCCTAAATGTGA and AGCAGTTGTGGCATCTCCTGATGAG with a FAM-labelled MGB RdRp Probe ATGCTTAGAATTATGGCCTCAC. The internal extraction control was detected using the MS2 forward primer TGGCACTACCCCTCTCCGTATTCACG, the MS2 reverse primer GTACGGGCGACCCCACGATGAC and a ROX-BHQ2 labelled MS2 probe CACATCGATAGATCAAGGTGCCTACAAGC. Amplification reactions and detection of PCR products were performed using the RotorgeneTM PCR instrument. A typical reaction contained $400 \mathrm{nM}$ of forward and reverse primers for the RdRp gene and 200nM of the MS2 internal control forward and reverse primer pair, along with $120 \mathrm{nM}$ of the RdRp and MS2 probes. The cycle conditions were as follows: $25^{\circ} \mathrm{C} 2$ mins, $50^{\circ} \mathrm{C} 15$ mins, $95^{\circ} \mathrm{C} 2$ mins followed by 45 cycles of $95^{\circ} \mathrm{C}$ and $60^{\circ} \mathrm{C}$. Samples that generated a CT value $\leq 36$, defined as 0.01 fluorescence units as per the RotorgeneTM manufacturer's instructions, were considered positive (roughly equivalent to 8 genome copies, the lower limit of detection for the assay). Where indicated, additional samples were tested in the clinical laboratory at the Royal London Hospital, London.

\section{Viral sequencing and bioinformatics}

Samples were analysed by Nanopore sequencing as part of the COG-UK sequencing project, following the ARTICnetwork V3 protocol (https://dx.doi.org/10.17504/protocols.io.bbmuik6w), and assembled using 
the ARTICnetwork assembly pipeline (https://artic.network/ncov-2019/ncov2019-bioinformaticssop.html). The accession numbers of the sequences included in this study are available in Table 5. Median genome depth of coverage was 1,346x. Consensus FASTA sequences were analysed after QC filtering, de-duplication and matching with metadata. Variants were initially assessed using the ARTICnetwork assembly pipeline VCF output files, with a SNP being called when $>50.1 \%$ frequency at a single base. These were then independently confirmed using iVar analysis (https://andersenlab.github.io/ivar/html), set to a quality threshold of Q20 and a minimum frequency of $10 \%$, with a SNP being called when $>50.1 \%$ of the reads at a particular position differed from the reference sequence, Wuhan-Hu-1 (GenBank Accession MN908947.3). The location of each SNP was examined to identify mutations in the $n s p 12$ gene, encoding RdRp (the target of remdesivir treatment). For the kinetic assessment, proportions of each variant as a fraction of all reads at their corresponding locations (minimum depth 20x) were plotted as a proportional stacked area chart using ggplot2 and RStudio.

\section{$\underline{\text { Intracellular cytokine staining }}$}

Cryopreserved PBMCs were rapidly thawed, washed in R10 media (RMPI-1640 + 10\% FBS $+1 \%$ Pen/Strep), and $10^{6}$ cells added to wells of a 96-well U-bottom plate. Cells were stimulated with $2 \mathrm{ug} / \mathrm{ml}$ overlapping $\mathrm{S} 1$ or $\mathrm{M}$ peptide pools, or left unstimulated, for $2 \mathrm{hr}$ at $37^{\circ} \mathrm{C}, 5 \% \mathrm{CO} 2$. Anti-CD107a-BV785 (1:100 dilution, clone H4A3), anti-CD28 (clone CD28.2) and anti-CD49d (clone R1-2) were added at $1 \mathrm{ug} / \mathrm{ml}$ to all wells at the time of peptide addition. After $2 \mathrm{hr}$, brefeldin A (5ug/ml) and monensin (2uM) were added, and cells were incubated at $37^{\circ} \mathrm{C}, 5 \% \mathrm{CO} 2$ for an additional $16 \mathrm{hr}$. After stimulation, cells were washed two times with FACS buffer (PBS $+1 \mathrm{mM}$ EDTA + 0.05\% BSA). Surface staining was performed at $4{ }^{\circ} \mathrm{C}$ for 30 mins. Cells were then washed two times in FACS buffer, and fixed and permeabilized at $4{ }^{\circ} \mathrm{C}$ for 30 mins using BD Cytofix/Cytoperm solution. Cells were then washed twice with $1 \times$ BD Perm/Wash buffer, and stained for intracellular markers at $4^{\circ} \mathrm{C}$ for 30 mins. Two further washes with $1 \times B D$ Perm/Wash buffer were performed and cells were stored in FACS buffer at $4^{\circ} \mathrm{C}$. Samples were acquired on a custom Cytek Aurora spectral analyzer (4 laser; UV, violet, blue, and red) using SpectroFlo v2.2. Data were analyzed using FlowJo v. 10.6.2 and Prism v. 8.3.0.

T cell proliferation assay

Freshly isolated PBMCs were labelled using CellTrace Violet (Invitrogen) and stimulated with peptide pools spanning the entire S, M, N and ORFs 3, 6, 7, and 8 SARS-CoV-2 proteins at $1 \mathrm{ug} / \mathrm{ml}$ of each overlapping peptide (OLP). Stimulation was done in RPMI media (Sigma) supplemented with $10 \% \mathrm{AB}$ serum (Sigma), $1 \%$ Pen/strep and $1 \% \mathrm{~L}$ glutamine for 7 days at $37^{\circ} \mathrm{C} .0 .1 \% \mathrm{DMSO}$, representative of dimethyl sulfoxide (DMSO) content in peptide stimulated wells, was used as negative control and $2 \mathrm{ug} / \mathrm{ml}$ of PHA (Sigma) served as positive control. On day $4,100 \mathrm{ul}$ of media was exchanged with 100ul of fresh media. On day 7, cells were washed using FACS wash buffer (Biolegend) and stained with L/D Near Infra red (Invitrogen), CD3 FITC (Biolegend), CD4 AF700 (BD Bioscience) and CD8 PECY7 (Biolegend). Samples were subsequently fixed and acquired on a BD LSR II. Data was analysed using flowjo v10.6.2. 
Serological Assessment

\section{Serological reactivity to SARS-CoV-2 spike and nucleocapsid proteins}

Recombinant SARS-CoV-2 nucleocapsid and spike proteins were covalently coupled to distinctive carboxylated bead sets (Luminex; Netherlands) to form a multiplex assay. For protein coupling, beads were first activated with 1-ethyl-3-[3-dimethylaminopropyl]carbodiimide hydrochloride (Thermo Fisher Scientific) in the presence of $\mathrm{N}$-hydroxysuccinimide (Thermo Fisher Scientific), according to the manufacturer's instructions, to form amine-reactive intermediates. The activated bead sets were incubated with the corresponding proteins at a concentration of $50 \mathrm{ug} / \mathrm{ml}$ in the reaction mixture for $3 \mathrm{hr}$ at room temperature on a rotator. Beads were washed and stored in a blocking buffer (10mM PBS, $1 \%$ BSA, $0.05 \%$ NaN3).

Coupled bead sets were incubated with patient or control sera at a dilution of 1/100 for $1 \mathrm{hr}$ in 96 -well filter plates (MultiScreenHTS; Millipore) at room temperature in the dark on a horizontal shaker. Fluids were aspirated with a vacuum manifold and beads were washed three times with $10 \mathrm{mM}$ PBS $/ 0.05 \%$ Tween-20. Beads were incubated for 30 mins with a PE-labeled anti-human IgG-Fc antibody (Leinco/Biotrend), washed as described above, and resuspended in 100 $\mu$ PBS/Tween-20. They were then analyzed on a Luminex analyzer (Luminex / R\&D Systems) using Exponent Software V31. Specific binding was reported as mean fluorescence intensities (MFI). Stored sera collected in the diagnostic immunology laboratory prior to November 2019 were used as healthy controls. Sera collected from patients with PCR-confirmed COVID-19 were used as positive controls.

\section{Neutralisation activity}

Cell lines: HEK293T (Lehner laboratory stocks) and HEK293T+ACE2 cells were cultured in Iscove's Modified Dulbecco's Media (Sigma) supplemented with 1\% GlutaMAXä, 10\% FCS, 100units/ml penicillin and $100 \mu \mathrm{g} / \mathrm{ml}$ streptomycin (all Thermo Fisher), at $37^{\circ} \mathrm{C}$ in $5 \% \mathrm{CO}_{2}$. HEK293T cells constitutively expressing angiotensin-converting enzyme 2 (HEK293T+ACE2 cells) were generated by transduction of wildtype HEK293T cells with a pHRSIN-ACE2-hygro ${ }^{\mathrm{R}}$ lentivirus and selected $48 \mathrm{~h}$ post-transduction using $100 \mu \mathrm{g} / \mathrm{mL}$ hygromycin B (Sigma). All cells were confirmed to be mycoplasma negative (MycoAlert, Lonza).

Plasmids: plasmid pCG1-SARS-CoV-2 D19 expressing humanised SARS-CoV-2 D19 spike was created by amplification of truncated spike from pCG1-SARS-2-S (a kind gift from M. Hoffmann, Infection Biology Unit, Leibniz Institute for Primate Research, Gottingen, Germany) using Phusion polymerase (NEB) and primer pair CoV2optFor (ttgtatcggatccaccatgttcgtgtttctggtgctgctg) and CoV2optD19rev (atcccgatctagatcagcagcagctgccacagctaca). The amplified product, lacking the C-terminal 19 amino acids, was digested and re-cloned into the PCG1 vector using BamHI-Xbal sites. pHRSIN-ACE2-hygro ${ }^{R}$ was produced by cloning into Kpnl-Xhol-digested pHRSIN-pSFFV MCS(+) pGK-Hygro using NEBuilder HiFi DNA assembly (NEB) of the angiotensin-converting enzyme 2 (ACE2) gene amplified from HepG2 mRNA, using Phusion polymerase (NEB) and primer pair ACE2-CDNA_Fwd 
(cgcccgggggggatccactaggtaccatgtcaagctcttcctggctcc) and ACE2_cDNA_Rev

(ctagagtcgcggccgctctactcgagctaaaaggaggtctgaacatcatcagtgttttg), following reverse transcription using Superscript III (Thermo Fisher) and oligo (dT) ${ }_{15}$ (Promega). pHRSIN-firefly luc-Puro ${ }^{\mathrm{R}}$ was produced by ligation of the BamHI-Notl-digested firefly luciferase cDNA into BamHI-Notl-digested pHRSIN pSFFVEmGFP pGK puro.

Virus production: pseudotyped lentiviral stocks were generated through the co-transfection of HEK293T cells with a lentiviral expression vector plus the packaging plasmid pCMV $\triangle$ R8.91 and either pMD.G (VSVG) or pCG1-SARS-CoV-2 D19 spike plasmid, using TransIT-293 transfection reagent (Mirus) according to the manufacturer's recommendations. Viral supernatants were harvested $48 \mathrm{hr}$ post-transfection and cell debris was removed with a 0.45 -um filter prior to use or freezing.

Neutralisation assay: pseudotyed pHRSIN-firefly luc-Puro ${ }^{R}$ lentivirus supernatants were pre-incubated with diluted serum or plasma samples at $37^{\circ} \mathrm{C}$ for 60 mins, before addition of $1.25 \times 10^{4}$ HEK293T cells to each well. Transduction efficiencies were quantified $48 \mathrm{hr}$ post-transduction by measuring firefly luciferase activity in cell lysates using Bright-Gloä luciferase assay system (Promega) and read in a CLARIOstar luminometer (BMG). Data were plotted using GraphPad Prism 8 (non-linear regression, (Inhibitor) vs. response - Variable slope, four parameters) and virus neutralising antibody titre was obtained by determining the dilution factor which gave a $50 \%$ reduction in firefly luciferase levels, compared to the no serum control wells.

\section{$\underline{\text { Complement assays }}$}

The complement components $\mathrm{C} 3 \mathrm{a}, \mathrm{C} 3 \mathrm{c}$ and $\mathrm{C} 5 \mathrm{a}$ were measured in plasma samples from patients with COVID-19 using solid-phase ELISA assays (Hycult Biotech) according to the manufacturer's instructions. TCC was measured using an in-house ELISA with the anti-TCC neo-epitope antibody, ae1 as capture.

\section{Data Availability}

Viral sequence data is available from COVID-19 Genomics UK and the GSAID. The accession numbers are detailed in Table 5.

\section{Declarations}

Competing Interests: E.J.M.T. is an employee of Hycult Biotechnology Ltd. All other authors declare no competing interests.

\section{Acknowledgements}

JEDT is supported by the MRC (RG95376 and MR/L006197/1). NJM is supported by the MRC (CSF MR/P008801/1) and NHSBT (WPA15-02). PJL is supported by the Wellcome Trust (PRF 210688/Z/18/Z). PK is a Wellcome Investigator (WT109965MA). KGCS is a Wellcome Investigator (200871/Z/16/Z). PAL and KGCS are additionally supported by the EU H2O20 research and innovation programme under grant 
agreement 668036 (RELENT). This work was supported by the NIHR Oxford Biomedical Research Centre, Oxford Immunology Network COVID-19 Response T cell Consortium and the NIHR Cambridge Biomedical Research Centre. The viral sequencing was funded by COVID-19 Genomics UK, which is supported by funding from the Medical Research Council part of UK Research and Innovation, the National Institute of Health Research, and Genome Research, operating as the Wellcome Sanger Institute.

\section{Author contributions}

M.S.B, J.B.G, C.N.F, J.P. and J.E.D.T. provided clinical care and monitoring during remdesivir treatment. M.S.B, J.B.G, C.N.F.,A.S., J.P., S. K-A, S.G, D.V.,M.H.,T.I.C., L.D., I.B.W., N.S., P.J.L., N.J.M. and J.E.D.T provided clinical care, clinical data collection and clinical data analysis. L.M.,A.Y., J.S., J.Q.,E.T., W.L.H, N.L. and I.G.G. conducted viral sequencing and analysis. N.M.P., S.B., A.O., W.M.Z., T.M., L.B., L.T., F.M., E.J.M.T, C-P.H., H.D.A., V.A.V., L. C-G., S.E.L., J.R.B., K.G.C.S., R.D., P.B.M., P.A.L., P.K., P.J.L., N.J.M. and J.E.D.T. carried out and supervised the laboratory immunological assessment. W.H.O., L.E., H.H., D.J.R. and N.J.M. provided convalescent plasma. M.S.B., P.J.L., N.J.M., and J.E.D.T. wrote the paper with input from all other authors. All authors contributed to the analysis of the presented results.

\section{References}

1. Wang, M. et al. Remdesivir and chloroquine effectively inhibit the recently emerged novel coronavirus (2019-nCoV) in vitro. Cell Res 30, 269-271 (2020).

2. Gordon, C.J. et al. Remdesivir is a direct-acting antiviral that inhibits RNA-dependent RNA polymerase from severe acute respiratory syndrome coronavirus 2 with high potency. $J$ Biol Chem 295, 67856797 (2020).

3. Choy, K.T. et al. Remdesivir, lopinavir, emetine, and homoharringtonine inhibit SARS-CoV-2 replication in vitro. Antiviral Res 178, 104786 (2020).

4. Williamson, B.N. et al. Clinical benefit of remdesivir in rhesus macaques infected with SARS-CoV-2. Nature (2020).

5. Wang, Y. et al. Remdesivir in adults with severe COVID-19: a randomised, double-blind, placebocontrolled, multicentre trial. Lancet 395, 1569-1578 (2020).

6. Beigel, J.H. et al. Remdesivir for the Treatment of Covid-19 - Preliminary Report. N Engl J Med (2020).

7. Huang, C. et al. Clinical features of patients infected with 2019 novel coronavirus in Wuhan, China. Lancet 395, 497-506 (2020).

8. Wang, D. et al. Clinical Characteristics of 138 Hospitalized Patients With 2019 Novel CoronavirusInfected Pneumonia in Wuhan, China. JAMA (2020). 
9. Bearden, D., Collett, M., Quan, P.L., Costa-Carvalho, B.T. \& Sullivan, K.E. Enteroviruses in X-Linked Agammaglobulinemia: Update on Epidemiology and Therapy. J Allergy Clin Immunol Pract 4, 10591065 (2016).

10. Galama, J.M., Vogels, M.T., Jansen, G.H., Gielen, M. \& Heessen, F.W. Antibodies against enteroviruses in intravenous Ig preparations: great variation in titres and poor correlation with the incidence of circulating serotypes. J Med Virol 53, 273-276 (1997).

11. Huang, K.H. et al. B-cell depletion reveals a role for antibodies in the control of chronic HIV-1 infection. Nat Commun 1, 102 (2010).

12. Lake-Bakaar, G. et al. Hepatitis C virus and alanine aminotransferase kinetics following B-lymphocyte depletion with rituximab: evidence for a significant role of humoral immunity in the control of viremia in chronic HCV liver disease. Blood 109, 845-846 (2007).

13. Bergthaler, A. et al. Impaired antibody response causes persistence of prototypic T cell-contained virus. PLoS Biol 7, e1000080 (2009).

14. Seiler, P. et al. Additive effect of neutralizing antibody and antiviral drug treatment in preventing virus escape and persistence. J Virol 74, 5896-5901 (2000).

15. Soresina, A. et al. Two X-linked agammaglobulinemia patients develop pneumonia as COVID-19 manifestation but recover. Pediatr Allergy Immunol (2020).

16. Quinti, I. et al. A possible role for B cells in COVID-19? Lesson from patients with agammaglobulinemia. J Allergy Clin Immunol (2020).

17. Grifoni, A. et al. Targets of T Cell Responses to SARS-CoV-2 Coronavirus in Humans with COVID-19 Disease and Unexposed Individuals. Cell (2020).

18. Li, C.K. et al. T cell responses to whole SARS coronavirus in humans. J Immunol 181, 5490-5500 (2008).

19. Zhao, J. et al. Recovery from the Middle East respiratory syndrome is associated with antibody and T-cell responses. Sci Immunol 2 (2017).

20. Almeida, J.R. et al. Superior control of HIV-1 replication by CD8+ T cells is reflected by their avidity, polyfunctionality, and clonal turnover. J Exp Med 204, 2473-2485 (2007).

21. Tay, M.Z., Poh, C.M., Renia, L., MacAry, P.A. \& Ng, L.F.P. The trinity of COVID-19: immunity, inflammation and intervention. Nat Rev Immunol 20, 363-374 (2020).

22. Zhang, L. et al. Antibody responses against SARS coronavirus are correlated with disease outcome of infected individuals. J Med Virol 78, 1-8 (2006). 
23. Zhao, J. et al. Antibody responses to SARS-CoV-2 in patients of novel coronavirus disease 2019. Clin Infect Dis (2020).

24. Gralinski, L.E. et al. Complement Activation Contributes to Severe Acute Respiratory Syndrome Coronavirus Pathogenesis. mBio 9 (2018).

25. Shen, B. et al. Proteomic and Metabolomic Characterization of COVID-19 Patient Sera. Cell (2020).

26. Magro, C. et al. Complement associated microvascular injury and thrombosis in the pathogenesis of severe COVID-19 infection: a report of five cases. Transl Res (2020).

27. Grein, J. et al. Compassionate Use of Remdesivir for Patients with Severe Covid-19. N Engl J Med (2020).

28. Liu, L. et al. Anti-spike IgG causes severe acute lung injury by skewing macrophage responses during acute SARS-CoV infection. JCI Insight 4 (2019).

29. Harvala, H. et al. Convalescent plasma therapy for the treatment of patients with COVID-19: Assessment of methods available for antibody detection and their correlation with neutralising antibody levels. medRxiv (2020).

\section{Tables}

Table 1 - Results from the clinical biochemistry laboratory, collated from readings at the indicated stages of the patient's illness 
Days after inpatient admission

\begin{tabular}{|c|c|c|c|c|}
\hline & $\begin{array}{l}\text { Pre- } \\
\text { Remdesivir: } \\
\text { Day 30-31 }\end{array}$ & $\begin{array}{l}\text { Post First Course } \\
\text { Remdesivir: Day 45- } \\
51\end{array}$ & $\begin{array}{l}\text { Second Course } \\
\text { Remdesivir: Day } \\
60\end{array}$ & $\begin{array}{l}\text { Post Second Course } \\
\text { Remdesivir: Day } 67\end{array}$ \\
\hline $\begin{array}{l}\text { Serum Sodium } \\
\text { (mmol/litre) }\end{array}$ & $\begin{array}{l}135(133- \\
146)\end{array}$ & 139 (133-146) & $134(133-146)$ & \\
\hline $\begin{array}{l}\text { Serum } \\
\text { Potassium } \\
\text { (mmol/litre) }\end{array}$ & $3.6(3.5-5.3)$ & $4.3(3.5-5.3)$ & $3.4(3.5-5.3)$ & \\
\hline $\begin{array}{l}\text { Serum Urea } \\
\text { (mmol/litre) }\end{array}$ & $3.8(2.5-7.8)$ & $2.7(2.5-7.8)$ & $2.1(2.5-7.8)$ & \\
\hline $\begin{array}{l}\text { Serum } \\
\text { Creatinine } \\
\text { ( } \mu \mathrm{mol} / \text { litre })\end{array}$ & $75(62-115)$ & $77(62-115)$ & $56(62-115)$ & \\
\hline $\begin{array}{l}\text { Albumin } \\
\text { (g/litre) }\end{array}$ & 28 (36-50) & & & \\
\hline $\begin{array}{l}\text { Total Bilirubin } \\
(\mu \mathrm{mol} / \text { litre })\end{array}$ & $9(0-20)$ & & $5(0-20)$ & \\
\hline $\begin{array}{l}\text { Alkaline } \\
\text { Phosphatase } \\
\text { (U/litre) }\end{array}$ & $66(30-130)$ & & 77 (30-130) & \\
\hline $\begin{array}{l}\text { Alanine } \\
\text { Transaminase } \\
\text { (U/litre) }\end{array}$ & $26(10-49)$ & & $13(10-49)$ & \\
\hline
\end{tabular}

Triglyceride $3.17(0.3-$

( $\mathrm{mmol} /$ litre) $1.8)$

Abnormal results are shown in bold. Numbers in parentheses indicate reference values of the corresponding measurements.

Table 2 - Results from the clinical haematology laboratory, collated from readings at the indicated stages of the patient's illness 
Days after inpatient admission

\begin{tabular}{|c|c|c|c|c|}
\hline & $\begin{array}{l}\text { Pre- } \\
\text { Remdesivir: } \\
\text { Day 30-31 }\end{array}$ & $\begin{array}{l}\text { Post First Course } \\
\text { Remdesivir: Day 45- } \\
51\end{array}$ & $\begin{array}{l}\text { Second Course } \\
\text { Remdesivir: Day } \\
60\end{array}$ & $\begin{array}{l}\text { Post Second Course } \\
\text { Remdesivir: Day } 67\end{array}$ \\
\hline D-Dimer (ng/ml) & $201(0-230)$ & & & \\
\hline $\begin{array}{l}\text { D-Dimer (ml/L } \\
\text { FEU) }\end{array}$ & & $0.66(0-0.5)$ & $0.77(0-0.5)$ & \\
\hline $\begin{array}{l}\text { Fibrinogen } \\
\text { (g/litre) }\end{array}$ & $\begin{array}{l}6.33(1.46- \\
3.33)\end{array}$ & $7.3(1.46-3.33)$ & $6.61(1.46-3.33)$ & \\
\hline Ferritin $(\mu \mathrm{g} / \mathrm{L})$ & $\begin{array}{l}1063.5(22- \\
322)\end{array}$ & $410(22-322)$ & $546(22-322)$ & \\
\hline $\begin{array}{l}\text { Lactate } \\
\text { dehydrogenase } \\
\text { (U/litre) }\end{array}$ & $\begin{array}{l}620(120- \\
246)\end{array}$ & & & \\
\hline $\begin{array}{l}\text { NT Pro BNP } \\
(\mathrm{pg} / \mathrm{ml})\end{array}$ & 12 & & & \\
\hline $\begin{array}{l}\text { High sensitivity } \\
\text { troponin } \\
\text { (ng/litre) }\end{array}$ & $5.8(0-58.1)$ & & & \\
\hline APTT (sec) & $\begin{array}{l}36.1(28.2- \\
36.6)\end{array}$ & & $28(28.2-36.6)$ & \\
\hline PT (sec) & $\begin{array}{l}15.6(10.8- \\
13.3)\end{array}$ & $11.2(10.8-13.3)$ & $12(10.8-13.3)$ & \\
\hline
\end{tabular}

Abnormal results are shown in bold. Numbers in parentheses indicate reference values of the corresponding measurements. APTT, activated partial thromboplastin time; PT, Prothrombin time.

Table 3 - Results from the clinical immunology laboratory, collated from readings at the indicated stages of the patient's illness 
Days after inpatient admission

\begin{tabular}{llll} 
Pre- & Post First Course & Second Course & Post Second Course \\
Remdesivir: & Remdesivir: Day 45- & $\begin{array}{l}\text { Remdesivir: Day } \\
\text { Day 30-31 }\end{array}$ & $\begin{array}{l}\text { Remdesivir: Day 67 } \\
\text { 51 }\end{array}$ \\
\hline
\end{tabular}

\begin{tabular}{|c|c|c|c|}
\hline IgG (g/litre) & $\begin{array}{l}9.6(6.34- \\
18.11)\end{array}$ & $13.8(6.34-18.11)$ & $16.5(6.34-18.11)$ \\
\hline IgA (g/litre) & $\begin{array}{l}<0.3(0.8- \\
2.8)\end{array}$ & $<0.05(0.8-2.8)$ & $<0.05(0.8-2.8)$ \\
\hline $\operatorname{lgM}$ (g/litre) & $\begin{array}{l}<0.2(0.5- \\
1.9)\end{array}$ & $<0.05(0.5-1.9)$ & $<0.05(0.5-1.9)$ \\
\hline
\end{tabular}

Complement C3

(g/litre)

$2.26(0.75-$

1.65)

Complement C4 $0.66(0.14$ -

(g/litre)

$0.54)$

$\begin{array}{ll}\text { Alternative } & >129(66- \\ \text { pathway AP100 } & 129) \\ (\%) & \end{array}$

$\begin{array}{ll}\text { Classical } & >911(392- \\ \text { pathway } \mathrm{CH} 100 & 1019)\end{array}$

$(\mathrm{U} / \mathrm{ml})$

TNF Alpha $\quad 15.65(0-5)$

$(\mathrm{pg} / \mathrm{ml})$

IL-1 beta $\quad 0.76(0-3.1)$

$(\mathrm{pg} / \mathrm{ml})$

$\mathrm{IL}-10(\mathrm{pg} / \mathrm{ml}) \quad 2.91(0-1)$

IFN-gamma $\quad 19.19(<10)$

$(\mathrm{pg} / \mathrm{ml})$

$\mathrm{IL}-6(\mathrm{pg} / \mathrm{ml}) \quad 31.8(0-2)$

$\underline{\mathrm{CD}}{ }^{ \pm} \underline{T \text { cells }}$

\begin{tabular}{|c|c|c|}
\hline 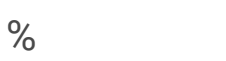 & 91 & 93 \\
\hline $\begin{array}{l}\text { Total }\left(\times 10^{9}\right. \\
\text { /litre) }\end{array}$ & $\begin{array}{l}1.21(0.7- \\
2.1)\end{array}$ & $1.61(0.7-2.1)$ \\
\hline
\end{tabular}

$\underline{\mathrm{CD}} 4^{ \pm} \underline{T \text { cells }}$

$\%$ 


\begin{tabular}{|c|c|c|}
\hline $\begin{array}{l}\text { Total }\left(x 10^{9}\right. \\
\text { /litre) }\end{array}$ & $\begin{array}{l}0.82(0.2- \\
1.4)\end{array}$ & 1.01 \\
\hline \multicolumn{3}{|c|}{$\underline{\mathrm{CD} 8}{ }^{ \pm} \underline{\mathrm{T} \text { cells }}$} \\
\hline$\%$ & 29 & 34 \\
\hline $\begin{array}{l}\text { Total (x109 } \\
\text { /litre) }\end{array}$ & $\begin{array}{l}0.39(0.2- \\
0.9)\end{array}$ & 0.58 \\
\hline \multicolumn{3}{|c|}{$\underline{\text { CD19 }} \pm \underline{B}$ cells } \\
\hline$\%$ & 0 & 0 \\
\hline $\begin{array}{l}\text { Total (x109 } \\
\text { /litre) }\end{array}$ & $0(0.1-0.5)$ & 0 \\
\hline \multicolumn{3}{|c|}{$\underline{\text { CD56 }}{ }^{ \pm}$NK cells } \\
\hline$\%$ & 9 & 6 \\
\hline $\begin{array}{l}\text { Total }\left(\times 10^{9}\right. \\
\text { /litre) }\end{array}$ & $\begin{array}{l}0.12(0.09- \\
0.6)\end{array}$ & 0.11 \\
\hline
\end{tabular}

Abnormal results are shown in bold. Numbers in parentheses indicate reference values of the corresponding measurements.

Table 4 - Results from the clinical microbiology laboratory, collated from readings at the indicated stages of the patient's illness 
Days after inpatient admission

\begin{tabular}{|lllll|}
\hline $\begin{array}{l}\text { Procalcitonin } \\
\text { (ng/ml) }\end{array}$ & $\begin{array}{l}\text { Pre- } \\
\text { Remdesivir: } \\
\text { Day 30-31 }\end{array}$ & $\begin{array}{l}\text { Post First Course } \\
\text { Remdesivir: Day 45- } \\
\mathbf{5 1}\end{array}$ & $\begin{array}{l}\text { Second Course } \\
\text { Remdesivir: Day } \\
\mathbf{6 0}\end{array}$ & $\begin{array}{l}\text { Post Second Course } \\
\text { Remdesivir: Day 67 }\end{array}$ \\
\hline HIV RNA & $\begin{array}{l}\text { Not } \\
\text { detected }\end{array}$ & $0.06(0-0.5)$ & \\
\hline Adenovirus DNA & $\begin{array}{l}\text { Not } \\
\text { detected }\end{array}$ & & \\
\hline $\begin{array}{l}\text { Human } \\
\text { metapneumovirus } \\
\text { RNA }\end{array}$ & $\begin{array}{l}\text { Not } \\
\text { detected }\end{array}$ & & \\
\hline $\begin{array}{l}\text { Influenza A } \\
\text { generic }\end{array}$ & $\begin{array}{l}\text { Not } \\
\text { detected }\end{array}$ & & \\
\hline Influenza B RNA & $\begin{array}{l}\text { Not } \\
\text { detected }\end{array}$ & \\
\hline $\begin{array}{l}\text { Parainfluenza } \\
\text { virus RNA }\end{array}$ & $\begin{array}{l}\text { Not } \\
\text { detected }\end{array}$ \\
\hline RSV RNA & $\begin{array}{l}\text { Not } \\
\text { detected }\end{array}$ \\
\hline Picornavirus RNA & $\begin{array}{l}\text { Not } \\
\text { detected }\end{array}$ \\
\hline
\end{tabular}

Abnormal results are shown in bold. Numbers in parentheses indicate reference values of the corresponding measurements.

Table 5 - Viral sequence accession numbers 


\begin{tabular}{|lll|}
\hline Virus name & COG-UK ID & GISAID Accession \\
\hline hCoV-19/England/CAMB-7FE20/2020 & CAMB-7FE20 & EPI_ISL_444421 \\
\hline hCoV-19/England/CAMB-82C3F /2020 & CAMB-82C3F & EPI_ISL_438672 \\
\hline hCoV-19/England/CAMB-1AC102/2020 & CAMB-1AC102 & EPI_ISL_444373 \\
\hline hCoV-19/England/CAMB-1AD7F0/2020 & CAMB-1AD7F0 & EPI_ISL_448012 \\
\hline hCoV-19/England/CAMB-1B2093/2020 & CAMB-1B2093 & EPI_ISL_453003 \\
\hline hCoV-19/England/CAMB-1B2CF9/2020 & CAMB-1B2CF9 & EPI_ISL_456720 \\
\hline hCoV-19/England/CAMB-1AC366/2020 & CAMB-1AC366 & N/A \\
\hline
\end{tabular}

Figures 

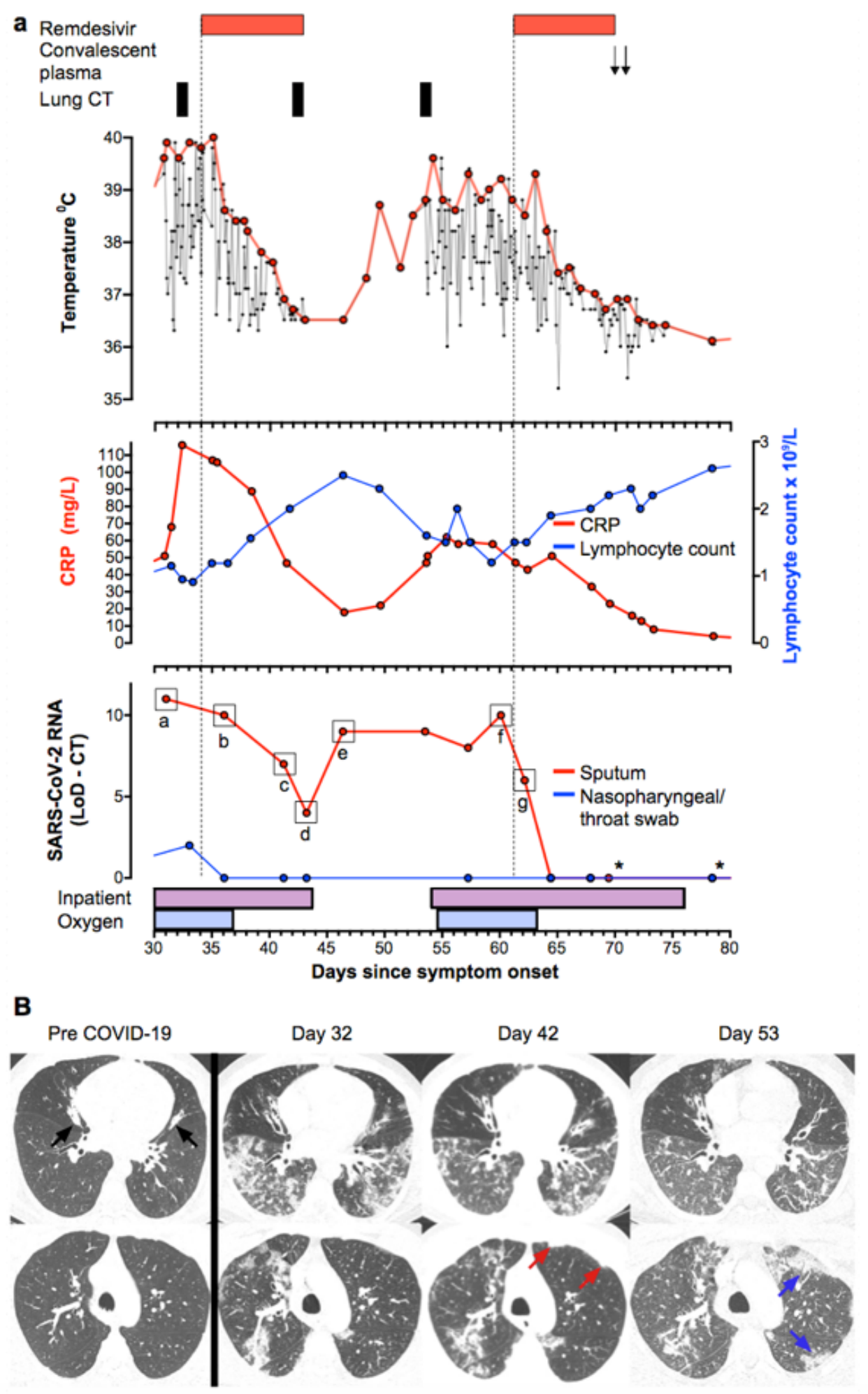

\section{Figure 1}

Clinical and virological assessment of the response to remdesivir. (a) Temperature, CRP, total lymphocyte count and Cycle-threshold (CT) value for viral RNA amplification from patient samples, deducted from the CT value at the limit of detection (LoD) of the assay, plotted by day since symptom onset, aligned with clinical interventions. Samples tested in the clinical laboratory at the Royal London Hospital are highlighted (*). Indicated viral isolates (a-g) were analysed by Nanopore sequencing (Supplementary 
Figure 2 and Table 5). (b) Computerised tomography (CT) images at the level of the inferior pulmonary veins (top) and aortic arch (bottom) obtained at indicated time points. Prior to COVID-19 infection, there is moderate bronchiectasis, mucoid impaction and small airway obstruction (black arrows) in the middle lobe and lingula, but the remainder of the lungs are clear (Pre-COVID-19). Shortly before the first course of remdesivir, extensive ground glass opacification and patchy consolidation with a lower lobe predominance is seen, together with perivascular consolidation in the right upper lobe (Day 32). 9 days after initiation of remdesivir treatment there is improvement in the lower zone ground glass opacity and consolidation, but persistent consolidation in the right upper lobe and mild progression of the left upper lobe subpleural consolidation (red arrows) (Day 42). 10 days after completion of the first course of remdesivir treatment, following relapse of symptoms and fever, there is further improvement in the lower zone ground glass opacification, but progressive subpleural consolidation, particularly in the left upper lobe (blue arrows) (Day 53).
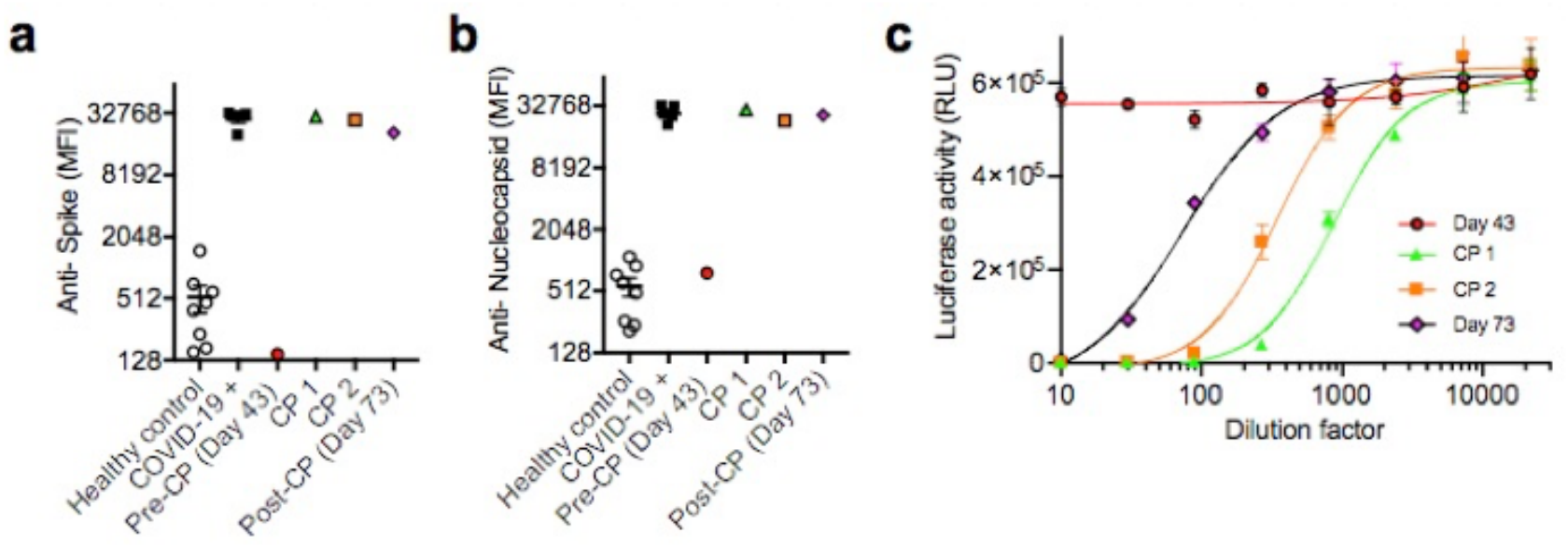

\section{Figure 2}

Kinetic assessment of the antigen-specific humoral responses. Serological reactivity to SARS-CoV-2 spike (a) and nucleocapsid (b) antigens of patient sera obtained at indicated time points, the first (CP 1) and second (CP 2) infusions of convalescent plasma (CP), sera from healthy controls and patients with PCRconfirmed COVID-19 (MFI, mean fluorescence intensity; Mean and SEM). (c) Neutralisation activity against SARS-CoV-2 spike-pseudotyped lentiviral particles of patient sera obtained at indicated time points, compared with the first (CP 1) and second (CP 2) infusions of convalescent plasma (CP). 

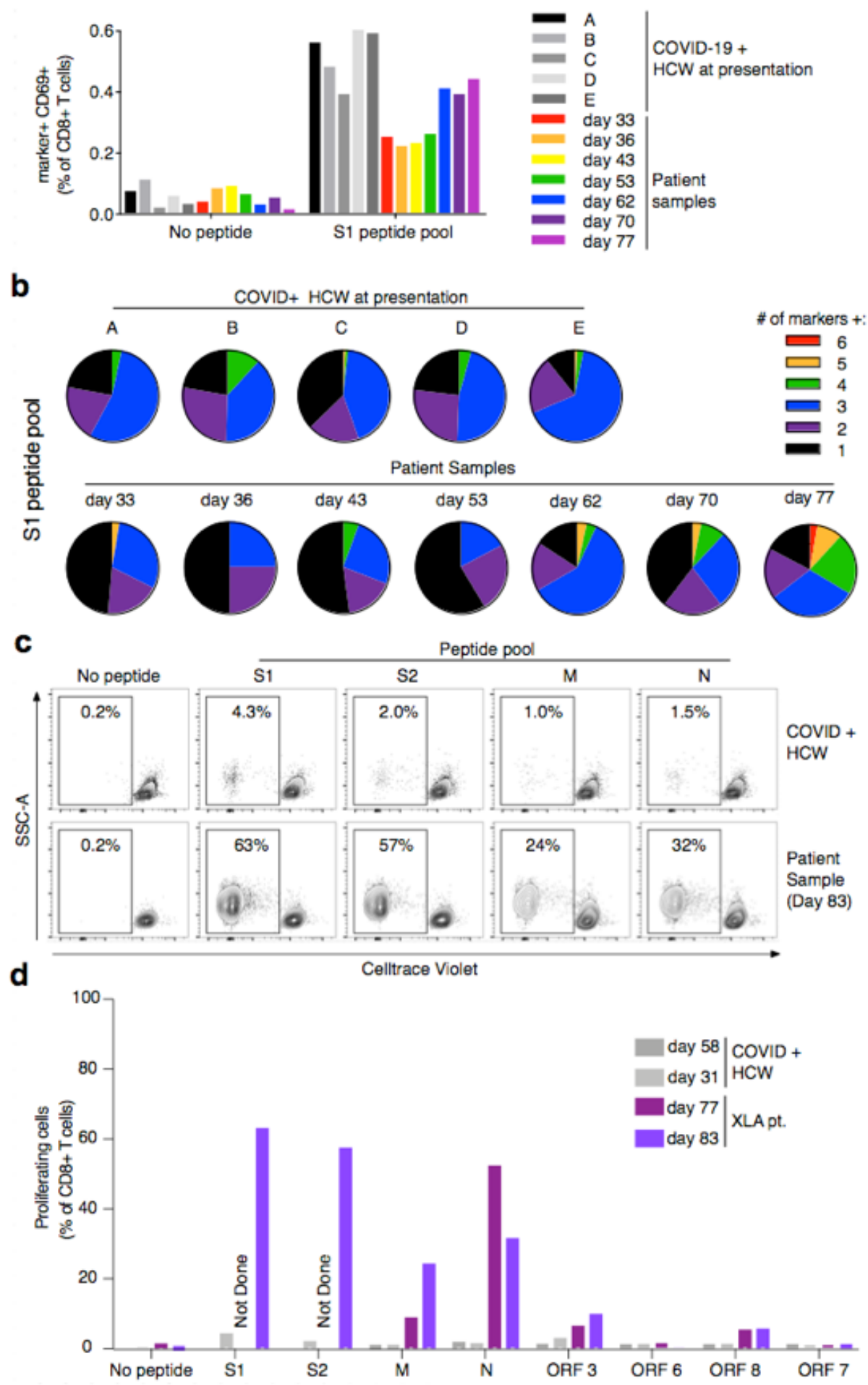

\section{Figure 3}

Kinetic assessment of the antigen-specific CD8+ T cell responses. (a) \% CD8+ T cells expressing activation makers after incubation \pm a peptide pool covering the SARS-CoV-2 S1 protein. Patient samples obtained at indicated time points are compared with $5 \mathrm{HCWs}$ with PCR-confirmed COVID-19 at presentation. (b). Number of activation markers expressed by S1-responsive CD8+ T cells from (a). Representative flow cytometry dot plots (c) and \% proliferating CD8+ T cells (d) after stimulation \pm 
peptide pools covering the indicated SARS-CoV-2 proteins. Patient samples are compared with $2 \mathrm{HCWs}$ with PCR-confirmed COVID-19 at indicated time points.
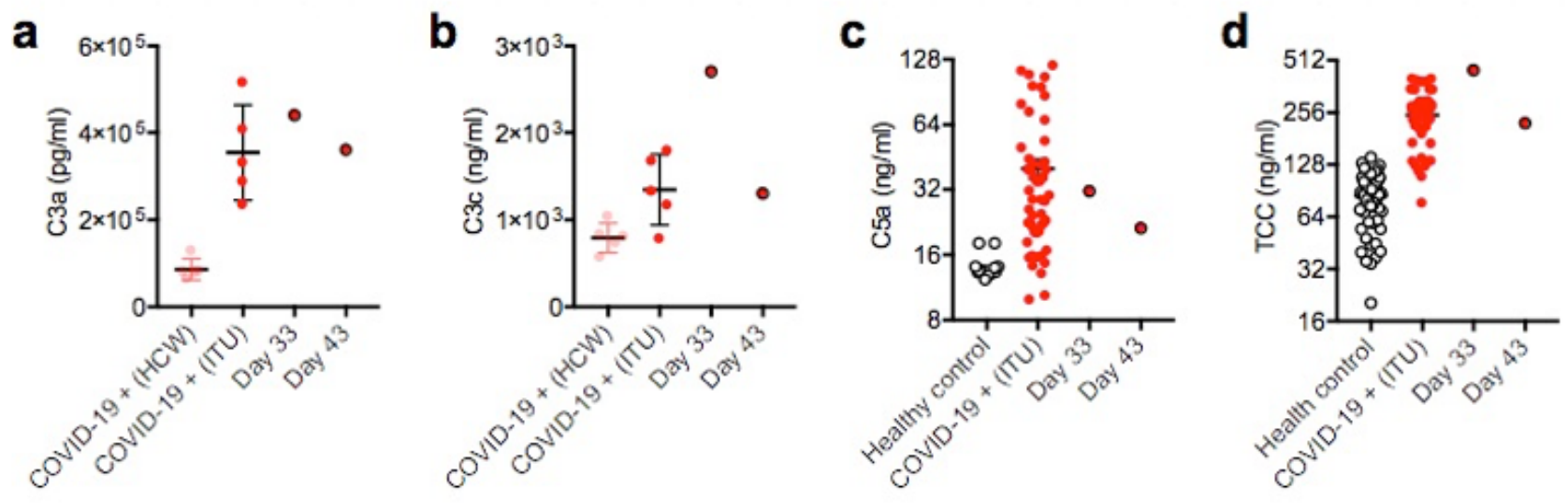

\section{Figure 4}

Assessment of complement activation. Concentrations of complement cleavage products C3a (a), C3c (b) and C5a (c), and circulating terminal complement complex (TCC) (d) at indicated time points, compared with healthy controls or patients with PCR-confirmed COVID-19 (healthcare workers, HCW or patients with severe disease admitted to the intensive therapy unit, ITU; Mean and SEM are shown). 